\title{
The Pixel Luminosity Telescope: a silicon sensor detector for luminosity measurement at CMS
}

\author{
Paul Lujan ${ }^{a, *}$ for the CMS Collaboration \\ ${ }^{a}$ Department of Physics, University of Canterbury, Private Bag 4800, Christchurch 8140, New Zealand \\ E-mail: paul.lujan@cern.ch
}

The Pixel Luminosity Telescope is a silicon pixel detector dedicated to luminosity measurement at the CMS experiment. It consists of 48 silicon sensor planes arranged into 16 "telescopes" of three planes each, with eight telescopes arranged around the beam pipe at either end of the CMS detector, outside the pixel endcap at a distance of approximately $1.75 \mathrm{~m}$ from the interaction point. The planes in a telescope are positioned such that a particle coming from the interaction point passing through a telescope will produce a hit in each of the three planes of the telescope. The instantaneous luminosity is measured from the rate of triple coincidences, using a special "fast-or" readout at the full bunch-crossing rate of $40 \mathrm{MHz}$, allowing for real-time, high-precision luminosity information to be provided to CMS and to the LHC. The full pixel information, including hit position and charge, is read out at a lower rate and can be used for studies of systematic effects in the measurement. We present the commissioning, calibration, operational history, and performance of the detector during Run 2 (2015-2018) of the LHC, together with lessons learned for future projects.

\footnotetext{
*** The European Physical Society Conference on High Energy Physics (EPS-HEP2021), ***

*** 26-30 July $2021 * * *$

*** Online conference, jointly organized by Universität Hamburg and the research center DESY ***
}

\footnotetext{
${ }^{*}$ Speaker
} 


\section{Introduction}

The Pixel Luminosity Telescope (PLT) [1, 2] is a dedicated system for measuring luminosity at the CMS experiment [3], using silicon pixel sensors. It was originally installed at the beginning of the 2015-2018 LHC running period (Run 2) by the CMS Beam Radiation, Instrumentation, and Luminosity (BRIL) project [4], and operated successfully throughout Run 2.

The PLT consists of 48 silicon sensors arranged into 16 "telescopes". Each telescope consists of three sensor planes, oriented perpendicular to the beam axis and spaced along the beam axis, such that particles originating from the CMS interaction point (IP) will pass through all three planes in the telescope. The rate of events where all three planes register a hit, called "triple coincidences", is then used as an estimator of the luminosity. The 16 telescopes are positioned with eight on either side of the CMS pixel endcaps (approximately $1.75 \mathrm{~m}$ from the IP), arranged in a circle around the beam pipe, at a pseudorapidity $|\eta| \approx 4.2$. Figure 1 shows the basic layout of a PLT telescope and the triple-coincidence operating principle.

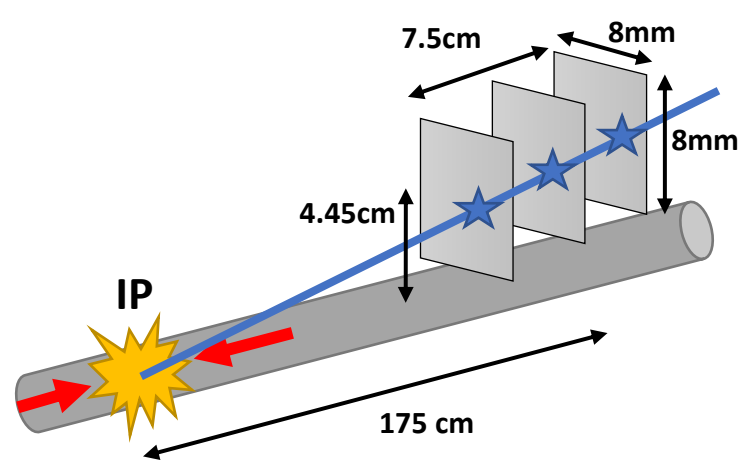

Figure 1: A sketch (not to scale) illustrating the basic operating principle of the PLT: a track originating from the CMS interaction point passing through a single PLT telescope will produce a triple coincidence. The center of the first plane is $4.45 \mathrm{~cm}$ from the beam axis, with the other two planes slightly farther away in the radial direction to match the slope of tracks coming from the IP.

The PLT uses the same sensors and readout chips (ROCs) as the CMS phase-0 pixel detector [3], which operated in CMS up to the end of 2016. These ROCs were designed with a special "fastor" readout mode, which was not used in the final CMS pixel detector, but was ideal for use in the PLT. The fast-or readout produces a single pulse if any pixels in a sensor register a hit over threshold during a single $25 \mathrm{~ns}$ LHC bunch crossing (BX). This signal is produced at the full $\mathrm{BX}$ frequency of $40 \mathrm{MHz}$ and thus can be used to measure triple coincidences to determine the per-bunch instantaneous luminosity. By operating at the full BX frequency, the resulting statistical precision is excellent even over short time intervals, and the triple coincidence requirement strongly reduces background from non-luminosity sources such as noise or "afterglow" from activated detector material. In addition to the fast-or readout, the regular full pixel readout is available with a dedicated trigger operating at a rate of approximately $3.3 \mathrm{kHz}$, providing information on hit positions and charge that can be used for further studies. 


\section{Luminosity measurement and calibration}

For a physics process with a given cross section $\sigma$, the rate of the process $R$ is related to the instantaneous luminosity $\mathcal{L}_{\text {inst }}$ by the relation $R=\mathcal{L}_{\text {inst }} \sigma$. In a similar manner, given the rate $R$ of an observable in a luminometer, we can write $R=\mathcal{L}_{\text {inst }} \sigma_{\text {vis }}$, where $\sigma_{\text {vis }}$, the "visible cross section", is the calibration constant, determined by the specific properties of the luminometer. Since the PLT measures each bunch individually, we can write $R_{i}=\mu_{i} f_{\mathrm{rev}}$, where $R_{i}$ is the per-bunch triple coincidence rate, $\mu_{i}$ is the average number of triple coincidences per bunch, and $f_{\text {rev }}$ is the LHC revolution frequency of $11.246 \mathrm{kHz}$. To avoid potential issues from multiple tracks not being individually resolved, $\mu_{i}$ is determined using the "zero counting" method, in which the fraction of events with no triple coincidences $f_{0}$ is measured, and $\mu_{i}$ is calculated by $\mu_{i}=-\ln f_{0}$, assuming the track counts follow a Poisson distribution.

The calibration constant $\sigma_{\text {vis }}$ is determined using the Van der Meer (VdM) method [5]. In the $\mathrm{VdM}$ method, a scan is performed in which the separation of the two beams is gradually varied first in the $x$ direction and then the $y$ direction, and the resulting measurement of the rate, normalized by the beam current, as a function of separation can be fit to determine the beam overlap width in each dimension. The resulting beam overlap width can then be used in conjunction with known beam parameters (such as the bunch charge) to compute the absolute luminosity. This knowledge can then be used to find $\sigma_{\text {vis }}$. The VdM scan is carried out in a fill with special beam conditions to maximize the precision of the $\mathrm{VdM}$ measurement and minimize systematic effects; the calibration derived from the VdM scan can then be used under regular running conditions, assuming a stable, linear detector response. For more information on the VdM scan procedure as used at CMS, see Refs. [6, 7]. Figure 2 shows an example of the PLT VdM scan data and the fitted curves from the 2017 VdM scan.

In practice, the relation $R=\mathcal{L}_{\text {inst }} \sigma_{\text {vis }}$ does not hold perfectly for a real luminometer, either due to a nonlinear response of the luminometer with respect to the luminosity, or due to a change in the detector response over time. In the PLT, these are principally caused by "accidentals" (triple coincidences not created by a genuine track) and by efficiency loss due to radiation damage in the sensors, respectively. The following section presents the procedure for measuring and correcting these effects.

\section{Linearity and efficiency corrections}

The principal method for measuring linearity and efficiency corrections in 2017-2018 was through emittance scans. These are scans performed by the LHC, usually at the beginning and end of each fill, to do a quick measurement of beam properties; they are similar to a VdM scan but much shorter, typically 7-9 scan points in each direction and only 10 seconds per scan point, so they can be performed in a few minutes. These scans can be analyzed in the same way as a standard VdM scan; although the statistical precision in the resulting beam width measurement is worse and the systematic effects are not as well measured, the large number of scans provides a large data sample to monitor relative efficiency changes over the course of a year. The scans at the beginning and end of each fill are performed at significantly different instantaneous luminosity, allowing for measurement of potential nonlinear behavior of the PLT. 

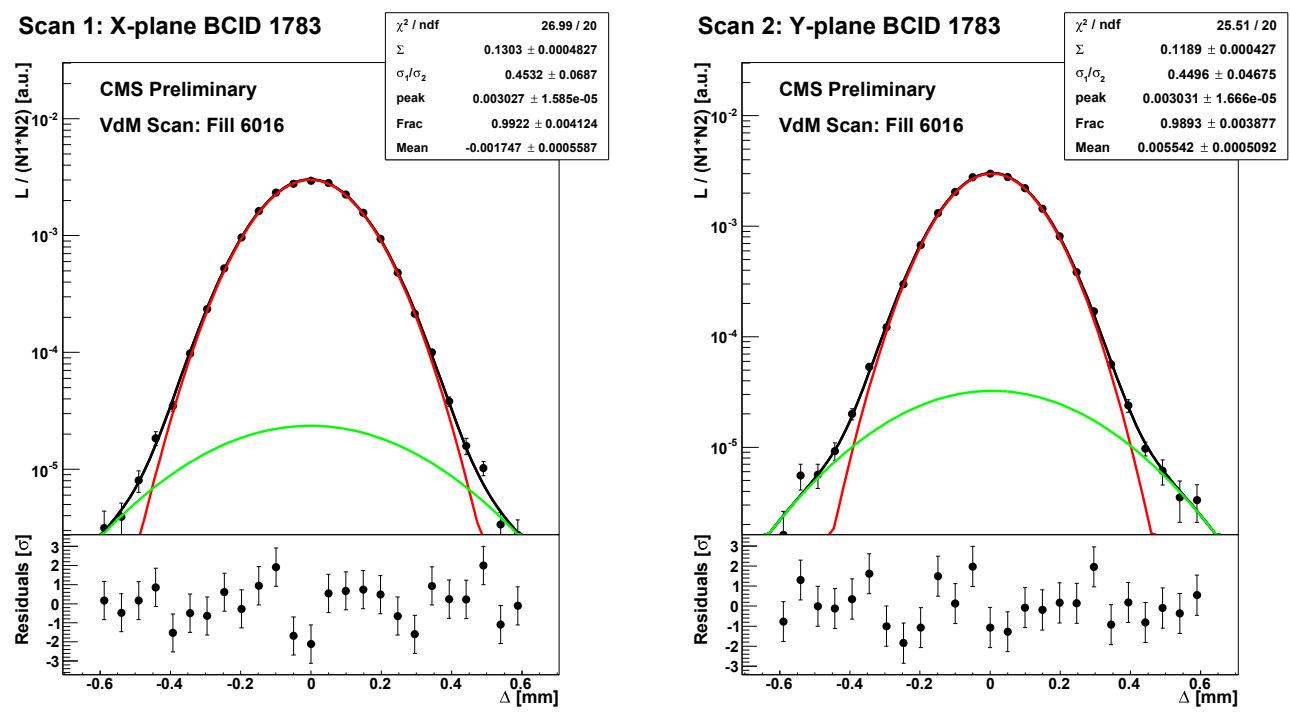

Figure 2: Normalized PLT rates and the resulting fitted double Gaussian scan curves (with the colored curves showing the two individual components) as a function of the beam separation $(\Delta)$ for a single bunch, for scans in the $x$ (left) and $y$ (right) direction in the 2017 VdM scan program.

Figure 3 shows the results from the emittance scans in 2017. The left plot shows the measured visible cross section as a function of the LHC fill number during the year; the loss of efficiency over time is clearly visible. The solid black lines indicate points at which the sensor bias high voltage was increased, which recovers some of the efficiency loss. The right plot shows the measured $\sigma_{\text {vis }}$ as a function of the single-bunch instantaneous luminosity (SBIL).

An alternate method for measuring the nonlinearity, and the one used in 2015-2016, is to assume that the principal source of the nonlinear response is "accidentals", cases where a triple coincidence is produced but there is no genuine track corresponding to a particle from the IP. This can be produced by, for example, two tracks which individually pass through only one or two planes of a telescope, but which together produce a triple coincidence. In order to measure these, we use the full pixel data and use the position information to reconstruct tracks. We look for events where a triple coincidence is recorded, but the resulting reconstructed track has either a slope value or a residual distance between the track and the hit more than $5 \sigma$ away from the average value. These triple coincidences are identified as accidentals, and the resulting rate as a function of SBIL is extracted. Figure 4 shows the resulting measured accidental percentage in 2015 and 2016, showing that the accidental rate shows a linear dependence on SBIL, with some systematic variation across the fills considered. The overall rates in 2016 are lower because the active area of the sensors was decreased at the beginning of 2016 to reduce the contribution from accidentals.

In a similar fashion, the efficiency can also be measured using the track data, by looking for events where there are two hits in two planes of a telescope that form a stub which extrapolates to 

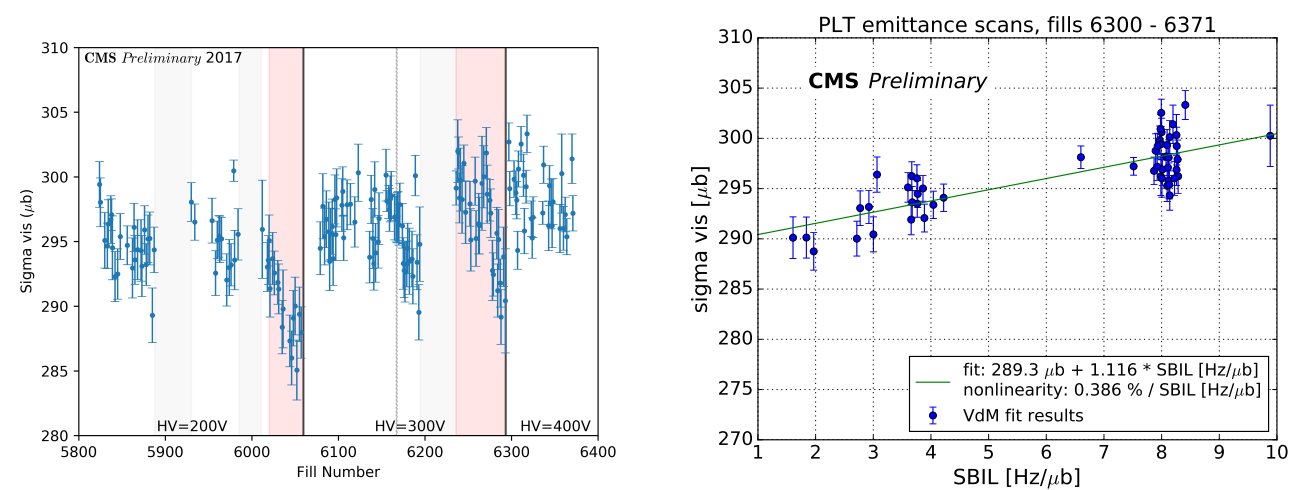

Figure 3: Measured visible cross section for the PLT using emittance scan data in 2017. Left: $\sigma_{\text {vis }}$ as a function of LHC fill number, showing loss of efficiency due to radiation damage. The solid black lines indicate times when the high voltage applied to the sensors was increased, and the shaded areas indicate periods of significant efficiency loss. Right: $\sigma_{\text {vis }}$ as a function of SBIL for some selected fills, showing slight nonlinear response in the PLT.
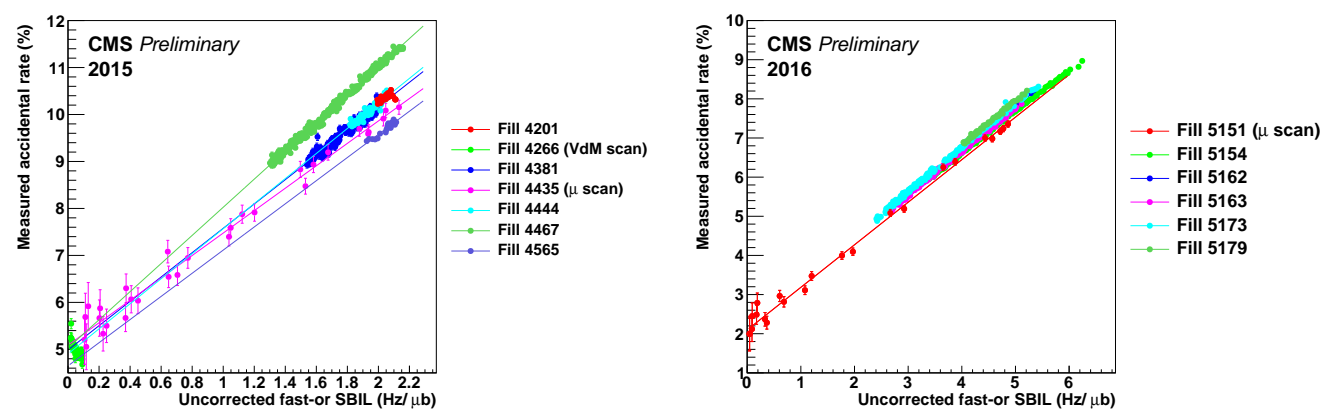

Figure 4: Measured PLT accidental rate, as a function of SBIL, for selected fills in 2015 (left) and 2016 (right). For 2015, a linear fit for each fill is shown; in 2016, for clarity, only the linear fit for fill 5151 is shown. The "mu scans" for each year are special fills where the luminosity is varied from its nominal physics value down to much lower values, allowing for measurement over a wide range of luminosity.

a location on the third plane, and looking for the fraction of events where that third hit is actually registered by the PLT. Figure 5 shows the results of this track efficiency measurement.

\section{Other studies}

It is also possible to perform a luminosity measurement using fully reconstructed tracks. This allows for direct rejection of accidental tracks, but the statistical precision is considerably lower than the fast-or measurement due to the lower readout rate for the full pixel data. Figure 6 (left) shows the resulting luminosity using reconstructed tracks, measured in 10-minute intervals over the course of an LHC fill, compared to the luminosity from the fast-or measurement and the forward hadron (HF) calorimeter luminosity. The agreement is generally good, although some remaining nonlinearity is visible. Because a higher trigger rate was used for VdM scans, the VdM scan analysis can be carried out using the track data, producing results that agree well with the fast-or measurement. 

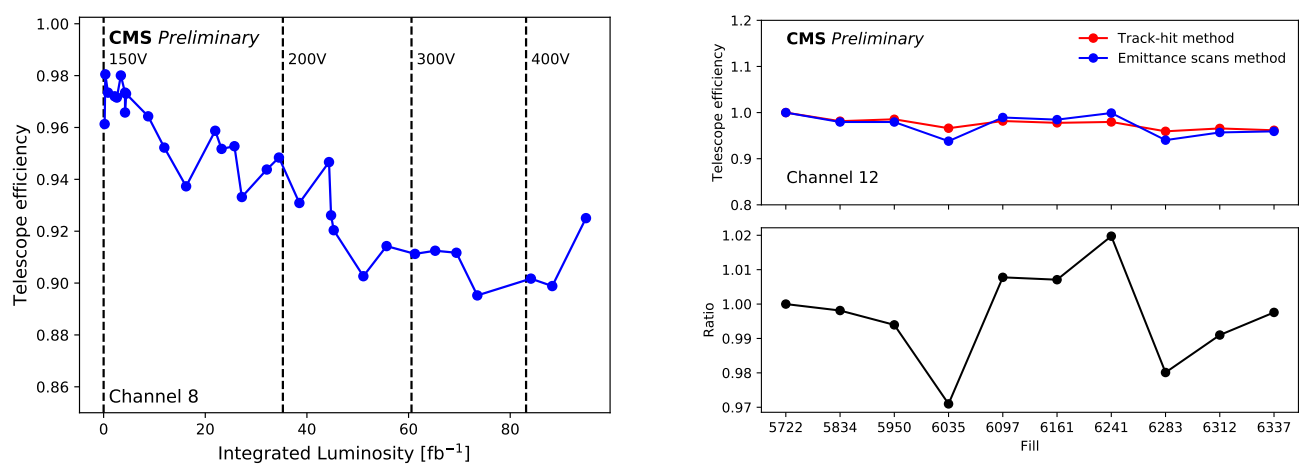

Figure 5: Left: Efficiency for a single PLT telescope (channel 8) as a function of integrated luminosity in 2015-2017. Right: Comparison of the efficiency from the track reconstruction method with the efficiency from emittance scans in 2017, showing good agreement between the two.
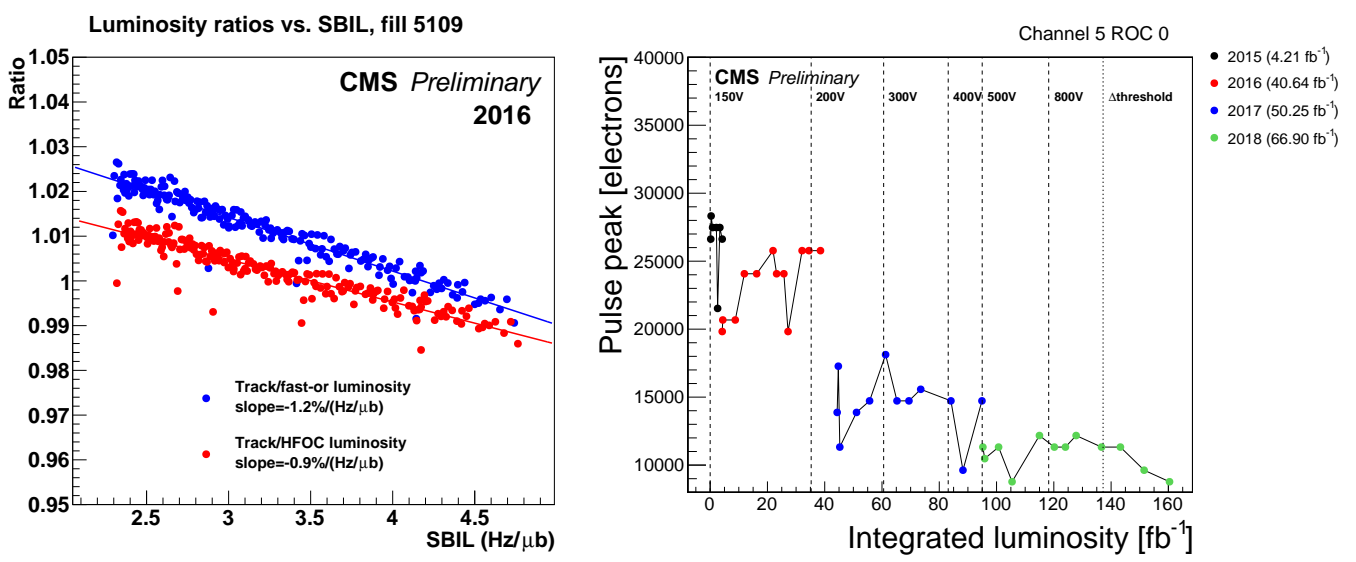

Figure 6: Left: Ratio of the luminosity obtained from track reconstruction vs. the fast-or luminosity (blue) and forward hadron calorimeter luminosity (HFOC, red) for a single LHC fill, shown as a function of SBIL. Right: Most probable value of the charge distribution as a function of integrated luminosity in 2015-2018 for a single PLT plane.

The full pixel data also includes information on the charge collected in the hit, which can also be used as a measure of radiation damage in the sensors. Figure 6 (right) shows the most probable value of the collected charge as a function of integrated luminosity in 2015-2018 for a single PLT plane, also showing the decline in the collected charge.

It is possible to perform automated quality monitoring of the pixel data by looking at the maps of the occupancy for each pixel in a sensor. For this algorithm, a set of 31 variables is constructed, such as the standard deviation of the number of hits, the number of rows or columns with different average numbers of hits, and so forth. The technique of $K$-means clustering is then used to divide the set of occupancy maps into good maps and maps exhibiting different kinds of issues, as shown in Fig. 7. This technique shows good promise for improving the PLT data quality in the upcoming LHC Run 3. 

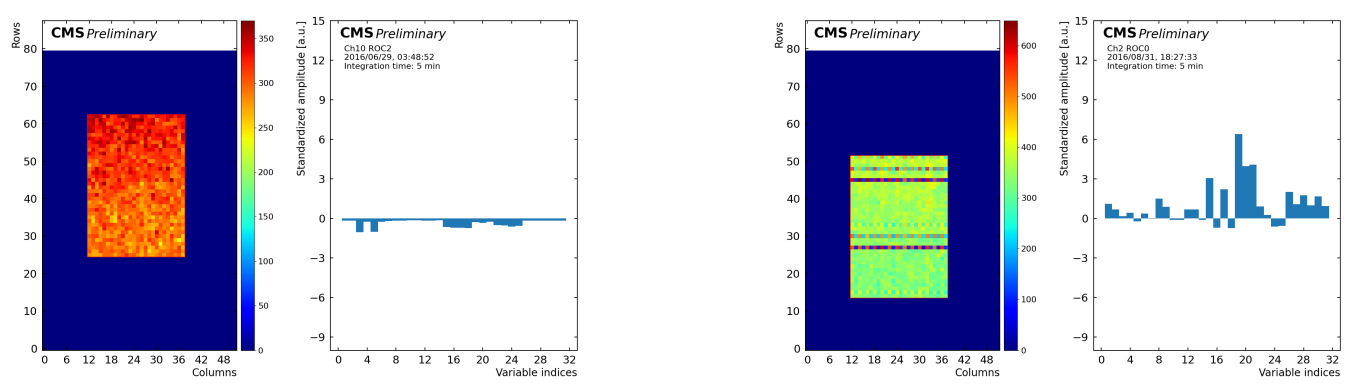

Figure 7: Examples of occupancy maps and the corresponding values of the variables used as input, for a period with good data (left) and a time period where the pixel locations in the raw data were not correctly decoded by the back-end hardware, resulting in an incorrect occupancy map (right).

\section{Summary}

The PLT operated successfully throughout the course of Run 2, providing high-precision bunchby-bunch luminosity both online, useful as fast feedback for LHC operations, and offline for physics analysis. The VdM method was used to provide an overall calibration for the PLT measurement, and analyses were developed to measure and correct for linearity and stability effects.

For the upcoming Run 3, a new copy of the PLT has been built and was installed in July 2021. It is currently undergoing commissioning and calibration during the LHC recommissioning in fall 2021, and will be ready for the beginning of physics operations in 2022. The Run 3 copy is largely identical to the Run 2 version, with a few small hardware changes. A second copy is under construction as a "hot spare" for installation during Run 3 in the event a partial or total replacement of the PLT is needed due to radiation damage. This will ensure that the PLT is able to continue to produce high-quality luminosity measurement throughout Run 3.

\section{References}

[1] A. Kornmayer on behalf of the CMS Collaboration, The CMS pixel luminosity telescope, Nucl. Instrum. Meth. A 824 (2016) 304.

[2] P. Lujan on behalf of the CMS Collaboration, Performance of the Pixel Luminosity Telescope for luminosity measurement at CMS during Run 2, PoS 314 (2017) 504.

[3] CMS collaboration, The CMS experiment at the CERN LHC, JINST 3 (2008) S08004.

[4] CMS collaboration, Upgrade of the CMS instrumentation for luminosity and machine induced background measurements, Nucl. Part. Phys. Proc. 273-275 (2016) 1147.

[5] S. van der Meer, Calibration of the effective beam height in the ISR, Technical Report CERN-ISR-PO-68-31 (1968).

[6] CMS collaboration, Precision luminosity measurement in proton-proton collisions at $\sqrt{s}=13$ TeV in 2015 and 2016 at CMS, Eur. Phys. J. C 81 (2021) 800 [2104.01927].

[7] CMS collaboration, CMS luminosity measurement for the 2018 data-taking period at $\sqrt{s}=13$ $\mathrm{TeV}$, CMS Physics Analysis Summary CMS-PAS-LUM-18-002 (2019). 